\title{
Doença renal crônica: informações de enfermagem aos envolvidos acerca da gravidade do consumo da carambola
}

\author{
Chronic kidney disease: nursing information for those involved about the gravity of the \\ consumption of a fruit
}

\section{Enfermedad renal crónica: información de enfermería para los involucrados sobre la gravedad del consumo de una fruta}

Silvia Rocha de Souza1*, Beatriz Maria dos Santos Santiago Ribeiro², Carolina Dominique dos Santos ${ }^{3}$.

\section{RESUMO}

Objetivo: Conhecer informações de enfermagem presente na literatura sobre o consumo de carambola em pacientes portadores de doença renal crônica. Revisão bibliográfica: Após a leitura dos artigos, foi possível elaborar três categorias temáticas: Compreendendo a doença renal crônica, Toxidade da carambola; Informações e orientações aos pacientes com Doença Renal Crônica. As informações sobre o consumo da fruta, em pacientes portadores de doença renal crônica, podem ser prevenidas as complicações da doença renal e dos demais agravos crônicos, percebe-se que é um desafio para o profissional de enfermagem aprimorar seus conhecimentos e empoderar-se do seu papel no cuidado e promoção de saúde. Ressalta-se a relevância em visar ações integradas para diminuição e evolução pelo consumo da carambola. Considerações finais: $O$ desenvolvimento deste estudo, abriu espaços para reflexões dos profissionais da saúde, uma vez que, geralmente não se observa a real necessidade em orientar sobre o consumo da fruta, cuja toxidade pode deixar o estado de saúde grave do paciente com doença renal crônica.

Palavras-chave: Doenças transmitidas por alimentos, Doença renal crônica, Enfermeiros e enfermeiras.

\begin{abstract}
Objective: To learn about nursing information in the literature on star fruit consumption in patients with chronic kidney disease. Bibliographic review: After reading the articles, it was possible to elaborate three thematic categories: Understanding chronic kidney disease, Toxicity of star fruit; Information and guidance for patients with Chronic Kidney Disease. Information on fruit consumption in patients with chronic kidney disease can prevent the complications of kidney disease and other chronic conditions, it is clear that it is a challenge for nursing professionals to improve their knowledge and empower themselves. its role in health care and promotion. The relevance of focusing on integrated actions to decrease and increase the consumption of star fruit is emphasized. Final considerations: The development of this study opened spaces for reflection by health professionals, since, generally, there is no real need to advise on the consumption of fruit, whose toxicity can leave the patient's severe health status with chronic kidney disease.
\end{abstract}

Keywords: Foodborne diseases, Chronic kidney disease, Nurses and nurses.

\section{RESUMEN}

Objetivo: Saber la información de enfermería presente en la literatura sobre el consumo de carambola en pacientes con enfermedad renal crónica. Revisión bibliográfica: Después de leer los artículos, fue posible elaborar tres categorías temáticas: comprensión de la enfermedad renal crónica, toxicidad de la fruta de estrella; Información y orientación para pacientes con enfermedad renal crónica. La información sobre el consumo de frutas en pacientes con enfermedad renal crónica puede prevenir las complicaciones de la

1 Unidade de Ensino Superior Ingá, Maringá - PA. *E-mail: silviaeadrean@hotmail.com

2 Universidade de São Paulo (USP), Ribeirão Preto - SP.

3 Universidade CESUMAR, Maringá - PA.

SUBMETIDO EM: 8/2020

ACEITO EM: 9/2020

PUBLICADO EM: 12/2020 
enfermedad renal y otras enfermedades crónicas, es claro que es un reto para el profesional de enfermería mejorar sus conocimientos y empoderarse. su papel en la atención y la promoción de la salud. Se enfatiza la relevancia de centrarse en acciones integradas para disminuir y aumentar el consumo de carambola. Consideraciones finales: El desarrollo de este estudio abrió espacios para la reflexión de los profesionales de la salud, ya que, en general, no hay una necesidad real de asesorar sobre el consumo de frutas, cuya toxicidad puede dejar el estado de salud grave del paciente con enfermedad renal crónica.

Palabras clave: Enfermedades transmitidas por los alimentos, Enfermedad renal crónica, Enfermeras y enfermeras.

\section{INTRODUÇÃO}

Os rins têm a função de filtrar o sangue, extrair os resíduos tóxicos produzidos nos tecidos do organismo, assim como a água e outras diversas substâncias que também produzem hormônios responsáveis em regular a pressão arterial, metabolismo ósseo e a produção de glóbulos vermelhos. Portanto, a perda da função renal resulta em uma série de problemas entre eles a Doença Renal Crônica (DRC) (MORSCH CMF e VERONESE FJV, 2011).

A perda progressiva e irreversível da função dos rins é denominada como DRC, sua causa mais frequente é doenças como diabete mellitus, hipertensão arterial e glomerulonefrites (MORSCH CMF e VERONESE FJV, 2011). Deve-se atentar-se a DRC, pois acontece silenciosamente até os seus estágios avançados (BASTOS RMR, et al., 2011)

As doenças crônicas são as principais causas de morbimortalidade no mundo (WORLD HEALTH ORGANIZATION, 2011). Além disso, doenças como a DRC no cenário mundial tem sido um grande desafio à saúde pública desse século, visto que há grandes implicações econômicas e sociais (BASTOS RMR, et al., 2009).

A carambola é uma fruta pertencente à família das oxalidaceae, atualmente vem sendo cultivada no Sudeste da Ásia, Malásia e em países tropicais como o Brasil. O seu tamanho de comprimento é de 6 a 13 centímetros, com 5 ou 6 proeminências longitudinais. Seu formato em fatias cortadas assemelha-se ao formato de uma estrela (MARGEN S,1992).

Quando essa fruta é ingerida por um paciente com DRC, pode surgir, inicialmente, sintomas neurológicos. Alguns trabalhos trouxeram esses sintomas descritos em pacientes renais crônicos submetidos a tratamentos dialítico, aparecendo com frequência soluços incoercíveis, vômitos, paresias e parestesias de membros superiores e inferiores com perda da força muscular, diversos distúrbios da consciência em graus variados; dentre eles agitação psicomotora, confusão mental, convulsões, que podem evoluir a óbito, principalmente em tratamento dialítico (NETO MM, et al.,1998; WANG JL, et al., 2000).

Diante do exposto anteriormente, salienta-se a relevância deste estudo, acreditando que em especial a equipe de enfermagem pode contribuir de modo significativo para a orientação ao portador de DRC. Já que a enfermagem é uma arte e uma ciência, cuja complexidade do cuidado vão além das assistências, o qual se deve apoiar nas orientações e acompanhamento dos clientes (RIBEIRO BMSS, et al., 2019).

Evidenciam-se a existência de poucos estudos sobre a toxidade da carambola no Brasil, bem como o encaminhamento de informações e orientações aos familiares e pacientes renais. Desse modo, há necessidade de informações para todos os envolvidos, pois podem contribuir para o planejamento de ações em conjunto com gestores e profissionais das instituições de saúde para serem implementadas ações que visam diminuir esse problema e assim propiciar um melhor bem-estar e qualidade de vida ao paciente renal. Ainda, essa pesquisa poderá colaborar com promoções de saúde.

Portanto, esse trabalho objetivou conhecer informações de enfermagem presente na literatura sobre 0 consumo de carambola em pacientes portadores de doença renal crônica. 


\section{REVISÃO BIBLIOGRÁFICA}

De acordo com o que foi levantado e com a literatura investigada é possível nesse estudo elaborar três categorias temáticas: Compreendendo a doença renal crônica, Toxidade da carambola e Informações e orientações aos pacientes com Doença Renal Crônica.

\section{Compreendendo doença renal crônica}

A avaliação da função renal consiste em utilizar a Taxa de Filtração Glomerular (TFG), que é definida como a competência dos rins em eliminar substância do sangue, se expressando como o volume de sangue que é inteiramente depurado em uma unidade de tempo. Essa medida é mais facilmente compreendida pelos médicos e pacientes, sendo um importante marcador mais sensível e específico das mudanças da função renal.

Sua definição é relacionada com a capacidade dos rins em eliminar uma substância do sangue e pode ser demonstrada pelo volume de sangue. Já o rim desenvolve a função de filtrar o sangue e eliminar os produtos finais do metabolismo proteico, preservando solutos específicos, como proteínas (particularmente albumina) e componentes celulares (KDIGO K, 2013).

A DCR é classificada pelos autores Morsch CMF e Veronese FJV (2011) em cinco estágios: O estágio 1 já há evidências de lesão renal, mas o paciente não apresenta sintomas, TFG maior que $90 \mathrm{ml} / \mathrm{min}$ e aumento de excreção de albumina em amostra de urina (>17 mg/l). No estágio 2: há dano renal leve e diminuição da função, com TFG entre $60-89 \mathrm{ml} / \mathrm{min}$.

A medida da creatinina no sangue apresenta-se normal, os rins nesse estágio ainda conseguem manter um controle razoável do meio interno (insuficiência renal leve). Já, o estágio 3: há sinais e sintomas discretos e o paciente se conserva clinicamente bem. TFG está entre $30-59 \mathrm{ml} / \mathrm{min}$, a creatinina no sangue mantém elevação (insuficiência renal moderada).

Em relação ao estágio 4, os sinais e sintomas são mais marcados uremia, anemia, falta de apetite e náuseas, pressão alta entre outros. Exames laboratoriais apresentam alteração marcando retenção de fósforo, queda do cálcio, alterações hormonais como deficiência de vitamina $D$, aumento do paratormônio, anemia de maior intensidade e retenção de ácidos produzidos (acidose). TFG está entre 15-29 $\mathrm{ml} / \mathrm{min}$ (insuficiência renal severa). Por fim, o estágio 5 há necessidade de diálise ou transplante de rim, TFG é menor que $15 \mathrm{ml} / \mathrm{min}$.

Os rins não conseguem manter o controle do meio interno e os distúrbios metabólicos são graves, aumentando o potássio presente no sangue, o qual pode desencadear arritmias cardíacas graves, retenção de ácidos, perda intensa do apetite, náuseas entre outros (KDIGO K, 2013; MORSCH CMF e VERONESE FJV, 2011).

A DRC vem crescendo diariamente em todo o mundo, anualmente sua incidência é de 8 a 16\%, sendo maior que o crescimento populacional geral. É possível controlar, prevenir ou retardar desfechos indesejados para portador de $\mathrm{DRC}$, isso se houver diagnóstico rápido, medidas nefro e cardioprotetoras implementadas imediatamente (GRASSMANN A, et al., 2005; CUSUMANO AM, et al, 2006; JHA V, et al, 2013).

A DRC como já mencionado é a perda progressiva e irreversível da função dos rins (BRASIL, 2006), são levado em consideração três elementos: elemento anatômico ou estrutural (marcadores de dano renal); elemento funcional baseado na TFG e elemento temporal (KDIGO K, 2013).

\section{Toxidade da carambola}

Já se passaram quase 40 anos da primeira descrição na Malásia, sobre intoxicação pela fruta da carambola em 1980, ainda não há necessidade de evidências cientificas sobre a natureza da toxina responsável pelos efeitos no sistema nervoso central (MUIR CK e LAM CK, 1980). Nessa época, nefrologistas e pesquisadores brasileiros contribuíram imensamente para trazer conhecimento clínico e farmacológico acercado do efeito da carambola em pacientes com DRC, o Jornal Brasileiro de Nefrologia foi o primeiro divulgador de informações dessa primeira descrição clínica (MARTIN, LC, et al,1993). 
Pesquisas experimentais utilizando ratos Wistar com insuficiência renal aguda assistidos apresentaram dois efeitos clínicos e eletroencefalográficos da intoxicação pela fruta notados em seres humanos. As variações dos sintomas diferem de paciente para pacientes, sendo explicados por respostas biológicas individuais e fatores como idade, quantidade da ingestão e da neurotoxina em cada porção, filtração glomerular residual entre outras, Diferenças também encontradas em animais de experimentação (GARCIA CN, et al., 2002).

Um estudo realizado com quatro pacientes que ingeriram carambola demonstrou que o principal sintoma decorrente desta ingestão é o soluço incoercível, mas que também apresentaram outros sintomas como convulsão tônico-clônica generalizada, vômitos, agitação psicomotora, distúrbios da consciência e de maior gravidade como convulsões iniciais que o paciente só estabilizou após 80 horas de hemodiálise.

O tempo de aparecimento dos sintomas após a ingestão da fruta foi de 02 a 12 horas variando de meia a seis unidades. A quantidade da ingestão interfere nos sintomas, pois quanto maior a ingestão maior a gravidade dos sintomas, assim podendo levar a óbito (NETO MM, et al., 2005).

No Hospital Metropolitano, localizado no Butantã, São Paulo, ocorreu um relato de caso, desenvolvido no ano de 2010 que alertou e informou sobre o caso de um cliente com idade de 56 anos, com as comorbidades instaladas de hipertensão, diabetes e insuficiência renal crônica, que ainda não havia necessidade de tratamento dialítico. Que dois dias antes da entrada teria ingerido quatro carambolas.

O quadro evolui muito rápido no período de 24 hora, no ambiente hospitalar> Logo após relato da ingestão da fruta, com queixa de mal-estar, cefaleia, náuseas e vômitos. Apresentou rebaixamento de nível de consciência e crises convulsivas reentrantes. Foi iniciado tratamento hemodialítico convencional, porém sem sucesso na melhoria do quadro clínico e com evolução a óbito quatro dias após a ingestão da carambola (MOREIRA FG, et al, 2010).

Outro caso clínico, que ocorreu em São Paulo, no Pronto Socorro HSPE. Paciente E.F.W.S., 44 anos, feminina, operadora de telemarketing, com queixa de diarreia havia quatro dias, acompanhada de náuseas, vômitos, dor abdominal em cólica e diminuição do volume urinário, sem outras queixas associadas. Referia que no dia anterior ao quadro, ingeriu suco de 20 carambolas e consumo de aproximadamente 30 frutas.

Negava a ingestão de outras medicações, além das habituais: enalapril, atenolol e levotiroxina. A paciente foi prontamente hidratada e suspenso o Inibidor da enzima de conversão da angiotensina (IECA), permanecendo anúrica, sugerindo que outro fator pudesse estar associado, a ingestão de carambola é a causa mais provável da lesão renal aguda (LRA). Assim, a carambola pode ser associada não só a complicações em portadores de DRC, mas também na LRA (SCARANELLO KL, et al, 2014).

Após detectar sintomas do consumo da carambola, o melhor tratamento ainda é o dialítico. Autores apontaram controvérsias com relação a duração, quantidade das seções, modalidade e o melhor momento para iniciá-lo. Mas, encontrou-se que o comum é a hemodiálise diária, com duração de 5 a 10 horas.

Ressalta-se que alguns casos mais graves, podem ocorrer confusão mental, e que a diálise contínua apresenta excelentes resultados, gerando um efeito rebote nos casos de intoxicação pela fruta. Já a diálise peritoneal, podem não ser o mais indicado para um tratamento eficaz, devido a relação como peso molecular da neurotoxina. Ainda, o tratamento da hemodiálise com carvão ativado, podem chegar com ótimos resultados, excelente resposta, rápida recuperação dos níveis de consciência e também diminuição do tempo de internação (WU MY, et al., 2007).

A fruta carambola possui dois tipos: com sabor mais azedo, que possui alto valor de ácido oxálico e as que possuem sabor mais doces, com teor menor, geralmente é produzida em países tropicais. Apresenta-se uma neurotoxina capaz de provocar grandes alterações neurológicas desde quadros leves a mais sérios em paciente com DRC. Além disso, essa neurotoxina mostra inibição sobre o sistema de condução Gabaérgico (CAROLINO ROG, 2002).

Portadores de DRC, ocorre a baixa excreção da toxina, portando eleva-se os níveis séricos, impedindo passagem pela barreira hematoencefálica e logo em seguida gera ação sobre o sistema nervoso central (WANG JL, et al., 2000). Já em pessoas sem nefropatias, a neurotoxina presente na fruta é absorvida com facilidade, distribuída e excretada pela via renal, sem danos ao organismo. 
Atualmente há veículos de divulgação sobre riscos da ingestão e recomendações presentes em publicações científicas e por outros meios de comunicação. No município de Jaú-SP, existe uma lei municipal e no estado do Mato Grosso do Sul uma lei estadual exigindo que em bares, lanchonetes e outros ambientes que oferecem alimentação transmitir a informação sobre os riscos do uso do consumo de carambola para doenças renais (BARRETTI P, 2015). Atitude essa que deveria ser expandida em todo território nacional.

Como mecanismo de ação da toxina presente na carambola, foi estudado pelo pesquisador Carolino ROG (2002) em sinaptosomas, verificando que é uma substância de baixo peso molecular, a mesma é solúvel em água e termoestável, sua estrutura está sob corrente investigação, cuja ação se refere a uma perturbação predominantemente do sistema Gabaérgico, por meio da alteração da ligação do GABA a seus receptores.

A literatura aponta sintomas leves como, por exemplo, soluços, vômitos, insônias; moderada paresias e parestesias de membros superiores e inferiores com perda da força muscular, diversos distúrbios da consciência em graus variados; agitação psicomotora, confusão mental leve; e graves convulsões mentais progredindo para estado de coma, instabilidade hemodinâmica evoluindo para hipotensão, choque e óbito, principalmente em tratamento dialítico (WANG JL, et al., 2000).

$\mathrm{O}$ autor Neto MM, et al., (2005) menciona que os pacientes devem ser alertados para não ingerirem carambola. Esse papel de advertência deve ser assumido pelos médicos e nutricionistas, e também por todos os envolvidos no tratamento dos pacientes portadores de DRC.

\section{Informações e orientações aos pacientes com Doença Renal Crônica}

Desse modo, é importante orientar o paciente, observando se as orientações ministradas foram entendidas pelo mesmo ou por familiares. De tal modo, o manejo de atividades que proporcionem a compreensão e gerem explicações é fundamental, assim garantindo uma boa orientação a ser ofertada.

Nessa perspectiva, a Enfermagem pode contribuir com a educação em saúde, realizando ações educativas, tais como a visita domiciliar, na internação do paciente renal, abordando sobre a toxidade do fruto que o paciente talvez desconheça, fornecendo-Ihes mais conhecimento e preparo para evitar ou intervir em complicações, diminuindo a demanda e custo nos serviços de saúde e, muitas vezes, salvando vidas.

Os pacientes com DRC em tratamento conservador necessitam se manter em boa condição clínica e compatível com o estágio de evolução de sua DRC, além de emocionalmente estável. Portanto faz-se necessário o uso de vários medicamentos, realização frequente de exames laboratoriais e normalmente seguir uma dieta bem restrita (CANHESTRO MR, et al., 2010). Ressalta-se que tratando da alimentação, o paciente deve estar ciente sobre não consumir a carambola, uma vez que sua toxidade pode trazer um prognóstico ruim.

Um estudo aborda a necessidade de um comportamento adequado em relação à adesão ao tratamento, tanto por parte dos pacientes como de seus familiares. Tal adesão é influenciada por diversos fatores relacionados ao paciente, a doença, ao tratamento, aos serviços e profissionais de saúde (CANHESTRO MR, et al., 2010)

Vale ressaltar que as Unidades de Saúde da Família, dentro da abordagem integral e resolutiva, são importantes para a disseminação de informações e orientações (FERREIRA RC, et al, 2009), são essenciais suas orientações sobre essa temática, visto que estão nos domicílios executando prevenções e promoções de saúde.

O trabalhador dessa área deve estar sempre por perto, identificando as necessidades do indivíduo, usando uma forma de comunicação continuada. Dessa forma, se estabelece como um instrumento de grande valia para estabelecer relações de confiança entre o profissional e o paciente (LEITE BS, 2012).

Os profissionais de enfermagem têm relação direta com o paciente, família e comunidade, possuem capacidade de desenvolver, de forma autônoma e em equipe atividades de promoção, proteção, prevenção e recuperação da saúde (OLIVEIRA I, 2006), lidam com pessoas que precisam ser cuidadas e requerem sua atenção, que vão além das habilidades técnicas (PRETO AV e PEDRÃO LJ, 2009). Dessa forma, são disseminadores de informações e orientações podendo contribuir significativamente com essa problemática. 
No trabalho interdisciplinar em equipe multiprofissional é plausível construir um processo de aprendizagem voltado ao cuidado integral à saúde das pessoas, a fim de promover o enriquecimento da qualidade de vida dos indivíduos (FERREIRA RC, et al., 2009).

Em especial, aquelas portadoras de DRC. Por isso é de extrema importância o trabalho multiprofissional, uma vez que se faz necessário a equipe conhecer seus pacientes a ponto de caracterizá-los, orientá-los acerca das necessidades para uma boa qualidade de vida e prognóstico bom com DRC.

Em relação a prevenção de complicações de doença renal e demais agravos crônicos, é necessário que o profissional de enfermagem aprimore seus conhecimentos e empodere-se do seu papel no cuidado e promoção de saúde visando à importância de ações integradas para diminuição e evolução pelo consumo da carambola.

Nesse contexto, destaca-se a importância da estratégia saúde da família, cuja assistência enfoca a promoção da saúde e a qualidade de vida da população, por meio de visitas domiciliárias e da transmissão de informações sobre os riscos inerentes as suas patologias, que na maioria dos casos são impossíveis de serem transmitidas durante as consultas nas UBS, orientações referentes à adoção de medidas de prevenção, envolvendo diagnóstico situacional.

No tocante ao acolhimento em serviços de saúde, o enfermeiro deve ficar atento as necessidades emocionais desses pacientes, pois estados de ansiedade, nervosismo e tristeza podem aumentar o consumo de alimentos. Sendo assim, um fator de risco a ingestão da carambola. Sugere-se a continuidade das pesquisas sobre essa temática, pois há poucos estudos sobre a toxidade da carambola, tendo como ponto inicial as problematizações o paciente renal crônico.

Enfatiza-se à conscientização da população em relação à toxidade, ações voltadas para contribuir com essa transmissão de conhecimento da sociedade em geral, o levantamento de informações que possam contribuir para que os profissionais, em especial, de enfermagem ampliem sua visão, desenvolvam competências e ações para prevenir agravos à saúde em virtude de tais acontecimentos.

Nesse estudo, contribui significativamente para que os profissionais de saúde estratégias para minimizar este o agravamento da DRC, decorrente do consume da fruta. Destaca-se que a presente revisão pode ser um grande subsídio para criação de protocolos futures relacionados as informações para clientes portadoras de DRC e sociedade em geral. Como limitação do estudo, abarca os poucos estudos, principalmente atuais em relação a temática.

Entretanto, acredita-se que o desenvolvimento do presente estudo, pode promover a reflexão dos profissionais da saúde, o qual muitas vezes não observa a real necessidade em orientar sobre o consumo dessa fruta, e que sua toxidade pode comprometer gravemente o paciente com DRC.

Por fim, acredita-se que os resultados do presente estudo possam contribuir para o estabelecimento de ações educativas voltadas para as orientações e informações ao paciente renal, para manter uma boa preservação de sua saúde.

\section{CONSIDERAÇÕES FINAIS}

Na literatura investigada, foi possível alcançar o objetivo proposto, por meio da descrição de três categorias temáticas: compreendendo a doença renal crônica, Toxidade da carambola e Informações e orientações aos pacientes com Doença Renal Crônica. Constatou que a carambola possui uma substância tóxica chamada caramboxina, que é a principal responsável pelos efeitos neurotóxicos e nefrotóxicos. Provocando soluços, vômitos, parestesias, confusão mental, crises convulsivas e, às vezes, levando o paciente crônico renal a morte. Por isso, é importante alertar aos pacientes com DRC a não consumirem carambola. Destaca-se o papel dos profissionais de saúde em especial, do enfermeiro para orientar, transmitir conhecimento a esse paciente, fornecendo todas as informações necessárias e esclarecendo dúvidas, respeitando o nível de entendimento de cada cliente. Ainda, se faz importante o preparo adequado da enfermagem, para orientação adequada da qualidade de vida e conscientização do cliente. 


\section{REFERÊNCIAS}

1. BRASIL. Ministério da Saúde. Secretaria de Atenção à Saúde. Departamento de Atenção Básica. Prevenção clínica de doenças cardiovasculares, cerebrovasculares e renais / Ministério da Saúde, Secretaria de Atenção à Saúde, Departamento de Atenção Básica. - Brasília, 2006. 56p.

2. BARRETTI P. Intoxicação por carambola em pacientes com doença renal crônica: da primeira descrição clínica à caramboxina. Universidade Estadual Paulista,2015.

3. BASTOS RMR, et al. Prevalência da doença renal crônica nos estágios 3, 4 e 5 em adultos. Revista da Associação Médica Brasileira, 2009; 55(1): 40-44.

4. CANHESTRO MR, et al. Conhecimento de pacientes e familiares sobre a doença renal crônica e seu tratamento conservador. Revista. Mineira de Enfermagem, jul./set., 2010,14(3): 335-344.

5. CAROLINO ROG. Atividade biológica e purificação parcial da neurotoxina da fruta Averrhoa carambola L. (Oxalidaceae). Dissertação de Mestrado apresentada ao Depto. de Bioquímica da Faculdade de Medicina de Ribeirão Preto - USP, 2002.

6. FERREIRARC, et al. Trabalho em equipe multiprofissional: a perspectiva dos residentes médicos em saúde da família. Revista Ciência e Saúde Coletiva. 2009; 14(1): 1421-8.

7. CUSUMANO AM, et al. The Latin American Dialysis and transplantation registry (RLDT) annual report 2004. Ethn Dis., 2006; 16(2): 2-10.

8. GARCIA CN, et al. A video-EEG study of the neurotoxic effects of star fruit (Averrhoa carambola) juice ingestion in animals with acute renal failure. Proceedings of the 39th European Renal Association and the European Dialysis and Transplantation Meetings, Copenhagen, Denmark. Nephrol Dial Transplant, 2002; 17: 89.

9. GRASSMANN A, et al. ESRD patients in 2004: global overview of patient numbers, treatment modalities and associated trends Nephrol Dial Transplant., 2005; 20: 2587-93.

10. JHA V, et al. Chronic kidney disease: global dimension and perspectives. Lancet, 2013; 382: 260-72.

11. KDIGO K. Disease Improving Global Outcomes CKD Work Group. Clinical practice guideline for the evaluation and management of chronic kidney disease. Kidney Int Suppl, 2013; 3: 1-150.

12. LEITE BS. A Relação entre os profissionais de enfermagem e pacientes terminais: a influência da comunicação. Revista Rede de Cuidados em Saúde. 2012; 6(1): 1-7.

13. MARTIN LC, et al. Soluço intratável desencadeado por ingestão de carambola (Averrhoacarambola) em portadores de insuficiência renal crônica. J Bras Nefrol 1993; 15: 92-4.

14. MARGEN S. The Wellness Encyclopedia of Food and Nutrition. New York: Health Letter Assoc, 1992. p. $271-2$.

15. MOREIRA FG, et al. Intoxicação por carambola em paciente com insuficiência renal crônica: relato de caso. Rev. bras. ter. Intensive, São Paulo. 2010; 22(4).

16. MORSCH CMF, VERONESE FJV. Doença renal crônica: definição e complicações. Rev HCPA, 2011

17. MUIR CK, LAM CK. Depressant action of averrhoa carambola. Med J Malaysia. 1980, 34(3):279-80.

18. NETO MM, et al.. Intoxication by star fruit (averrhoa carambola) in six dialysis patients? (Preliminaryreport). Nephrol Dial Transpl,1998;13:570-2.

19. NETO M, et al. Intoxicação por carambola (averrhoa carambola) em quatro pacientes renais crônicos pré-dialíticos e revisão da literatura. J Bras Nefrol, 4,2005, 228-232 p.

20. OLIVEIRA I. O acolhimento de familiares de pacientes internados em UTI: a tecnologia de grupo como estratégia para o cuidado de enfermagem [thesis]. Brasília: Universidade de Brasília; 2006. 219 p.

21. POLIT DS, et al. Compreensão do delineamento da pesquisa qualitativa. Fundamentos da pesquisa em enfermagem: métodos avaliação e utilização. 5. ed. Porto Alegre: Artmed; 2004. p.199-221.

22. PRETO AV, PEDRÃO LJ. O estresse entre enfermeiros que atuam em Unidade de Terapia Intensiva. Revista da Escola de Enfermagem da Universidade de São Paulo, 2009; 43(4): 841-848.

23. RIBEIRO BMSS, et al. Enfermagem do trabalho na construção civil: contribuições à luz da teoria da adaptação de Roy. Revista Brasileira de Medicina do Trabalho.2019; 17(2): 260-267

24. SCARANELLO KL, et al. Carambola como causa de lesão renal aguda. J. Bras. Nefrol. São Paulo, 2014; 36(2).

25. WANG JL, et al. (In Chinese) Status epilepticus in two patients with chronic renal failure after ingestion of star fruit. Kidney Dial 2000; 8:166-9.

26. WORLD HEALTH ORGANIZATION. Global status report on noncommunicable diseases 2010. Geneva: 2011.

27. WU MY, et al. Hemoperfusion as na effective alternative therapy for star fruit into, 2007. 